\title{
PONCELET'S PORISM IN TRANSFORMATION GROUP FRAMEWORK
}

\author{
Waldemar Cieślak' ${ }^{1}$, Halina Felińska', Janusz Szuster' ${ }^{1}$, Witold Mozgawa² \\ 1 Department of Applied Mathematics, Lublin University of Technology, Nadbystrzycka 38, 20-618 Lublin, \\ Poland. \\ 2 Institute of Mathematics, Maria Curie-Sklodowska University, M. Curie-Skłodowskiej 1 Sq., 20-031 Lublin, \\ Poland, e-mail: mozgawa@poczta.umcs.lublin.pl
}

Received: 2014.09.15

Accepted: 2014.10.25

Published: 2014.12.01

\begin{abstract}
In this paper we introduce a transformation group connected with Poncelet's porism. Several open questions following from our considerations end the paper. The aim of this paper is to give a new approach to find an algebraic Fuss-type formula for all natural $n>2$. Developed method may be applied to investigations of Poncelet's porism.
\end{abstract}

Keywords: transformation group, Poncelet's porism.

MSC2010: 53A04, 51M15, 37E99

\section{INTRODUCTION}

With each pair of circles we associate three positive numbers, namely their radii and the distance between their centers. If a pair of circles forms an annulus then their radii $r, R$ and the distance $a$ between their centers satisfy the inequality $r+a<R$. We consider a class of all annuli with a property of the Poncelet porism, see [3]. This class is divided onto classes of annuli with a property of the Poncelet porism for a fixed natural number $n$. In a case $n=3$ Poncelet's porism means that if we can circuminscribe in an annulus (inscribe in the outer circle and describe on the inner circle) one triangle then by each point of the annulus passes a circuminscribed triangle. For $n=3$ we have the following equality $a^{2}=R^{2}-2 r R$, see [2]. Similarly, for $n=4$ we consider circuminscribed quadrangles and so on. For $n=4$ the following equality $\left(R^{2}-a^{2}\right)^{2}=2 r^{2}\left(a^{2}+R^{2}\right)$ holds, see [2]. A compact algebraic formula connecting $R, r$ and $a$ for arbitrary $n$ does not exist. There exists an integral formula connecting $R, r$ and $a$ for arbitrary $n$ given for example by Jacobi, see $[6,2]$. Note, that simple formula involving elliptic integrals is given in [5].

\section{A TRANSFORMATION GROUP}

Let $\mathbb{R}_{+}=(0,+\infty)$. We consider the multiplicative group $\left(\mathbb{R}_{+},{ }^{\prime}\right)$ and the set

$$
\mathbb{M}=\left\{(R, r, a): R, r, a \in \mathbb{R}_{+}, r+a<R\right\} .(2.1)
$$

We define a function $f: \mathbb{R}_{+} \times \mathbb{M} \rightarrow \mathbb{M}$ by the formula

$$
f(\xi,(R, r, a))=((1-\xi) r+\xi R, r, \xi a) .
$$

Note that the function $f$ is well defined. It is easy to verify that for each $\xi, \eta \in \mathbb{R}_{+}$and $(R, r, a) \in \mathbb{M}_{\text {we have }}$

$$
f_{\xi} \circ f_{\eta}=f_{\xi \eta}
$$

and

$$
f_{1}=\mathrm{id},
$$

where

$$
f_{\xi}(R, r, a)=f(\xi,(R, r, a)) .
$$

Thus, we have

Theorem 2.1. The group $\left(\mathbb{R}_{+}, \cdot\right)$ acts on $\mathbb{M}$ from the left. 


\section{PONCELET'S PORISM FOR TRIANGLES}

Let us fix two circles $C_{r}, C_{R}$ satisfying the condition $r+a<R$, where $a$ denotes the distance between their centers. If the annulus $C_{r} C_{R}$ has the property of the Poncelet porism for $n=3$ then the Euler triangle formula is satisfied, namely

$$
a^{2}=R^{2}-2 r R
$$

Let

$$
\mathbb{M}_{3}=\left\{(R, r, a) \in \mathbb{M}: a^{2}=R^{2}-2 r R\right\}
$$

We put $t=\frac{R}{r}$. The equality (3.1) implies that $\left(\frac{a}{r}\right)^{2}=t^{2}-2 t$. For a fixed $t>2$ we define the set

$$
\mathbb{M}_{3}(t)=\left\{\left(r t, r, r \sqrt{t^{2}-2 t}\right) \in \mathbb{M}: r>0\right\} .
$$

Let

$$
\mathbb{M}_{3}=\bigcup_{t>2} \mathbb{M}_{3}(t)
$$

Theorem 3.1. If $(R, r, a) \in \mathbb{M}_{3}$ and $\xi \in(0,1)$ then there exists $\rho \in(0,1)$ such that

$$
((1-\xi) r t+\xi R, \rho r, \xi a) \in \mathbb{M}_{3} .
$$

Proof. Let an element $((1-\xi) r t+\xi R, \rho r, \xi a)$ belongs to $\mathbb{M}_{3}$. Then it satisfies the equality (3.1). Hence we have

$$
\rho=\frac{2 \xi(R-r)+\left(1+\xi^{2}\right) r}{2 \xi(R-r)+2 r} .
$$

It is easy to see from the expression (3.6) that $\rho \in(0,1)$ for $\xi \in(0,1)$. It follows from (3.6) that

$$
\begin{gathered}
((1-\xi) r t+\xi R, \rho r, \xi a)=r \rho\left(\frac{(1-\xi) r+\xi R}{r \rho}, 1, \frac{\xi a}{r \rho}\right) \\
=r \frac{2 \xi(t-1)+1+\xi^{2}}{2 \xi(t-1)+2}\left(2 \frac{(\xi(t-1)+1)^{2}}{2 \xi(t-1)+1+\xi^{2}}, 1, \xi \sqrt{t^{2}-2 t} \frac{2 \xi(t-1)+2}{2 \xi(t-1)+1+\xi^{2}}\right) \\
=r_{\xi}\left(t_{\xi}, 1, \sqrt{t_{\xi}^{2}-2 t_{\xi}}\right),
\end{gathered}
$$

where

$$
r_{\xi}=r \frac{2 \xi(t-1)+1+\xi^{2}}{2 \xi(t-1)+2}
$$

and

$$
t_{\xi}=2 \frac{(\xi(t-1)+1)^{2}}{2 \xi(t-1)+1+\xi^{2}}
$$

For a fixed $\xi \in(0,1)$ Theorem 3.1 generates the transformation $F_{\xi}: \mathbb{M}_{3} \rightarrow \mathbb{M}_{3}$ given by the formula

$$
F_{\xi}\left(r\left(t, 1, \sqrt{t^{2}-2 t}\right)\right)=r_{\xi}\left(t_{\xi}, 1, \sqrt{t_{\xi}^{2}-2 t_{\xi}}\right) .
$$

Theorem 3.2. The transformation $F_{\xi}$ is a surjection.

Proof. For an arbitrary fixed $\left(R_{0}, r_{0}, a_{0}\right)=r_{0}\left(t_{0}, 1, \sqrt{t_{0}^{2}-2 t_{0}}\right) \in \mathbb{M}_{3}$ and $\xi>0$ we may write $F_{\xi}(R, r, a)=\left(R_{0}, r_{0}, a_{0}\right)$, or

$$
F_{\xi}\left(r t, r, r \sqrt{t^{2}-2 t}\right)=\rho r\left(\frac{(1-\xi) r+\xi r t}{\rho r}, 1, \frac{\xi r \sqrt{t^{2}-2 t}}{\rho r}\right)=r_{0}\left(t_{0}, 1, \sqrt{t_{0}^{2}-2 t_{0}}\right) \text {, }
$$


where $\rho$ is given by (3.6). The above equations lead us to

$$
\begin{gathered}
t=1+\frac{t_{0}-2}{2 \xi}+\frac{1}{2 \xi} \sqrt{t_{0}^{2}-2\left(1-\xi^{2}\right) t_{0}}, \\
r=r_{0} \frac{2 t_{0}}{t_{0}+\sqrt{t_{0}^{2}+2 t_{0}\left(\xi^{2}-1\right)}} .
\end{gathered}
$$

It is easy to verify that $t>2$ and $r>0$.

\section{PONCELET'S PORISM FOR QUADRANGLES}

Let

$$
\mathbb{M}_{4}=\left\{(R, r, a) \in \mathbb{M}:\left(R^{2}-a^{2}\right)^{2}=2 r^{2}\left(a^{2}+R^{2}\right)\right\}
$$

From the condition in (4.1) for $t=\frac{R}{r}$ we obtain $\left(\frac{a}{r}\right)^{2}=t^{2}+1-\sqrt{4 t^{2}+1}$. Hence we have $t>\sqrt{2}$. For a fixed $t>\sqrt{2}$ we define the set

$$
\mathbb{M}_{4}(t)=\left\{\left(r t, r, r \sqrt{t^{2}+1-\sqrt{4 t^{2}+1}}\right): r>0\right\}
$$

Obviously, we have

$$
\mathbb{M}_{4}=\bigcup_{t>\sqrt{2}} \mathbb{M}_{4}(t)
$$

Theorem 4.1. If $(R, r, a) \in \mathbb{M}_{4}$ and $\xi \in(0,1)$ then there exists $\rho \in(0,1)$ such that

$$
((1-\xi) r t+\xi R, \rho r, \xi a) \in \mathbb{M}_{4} .
$$

Proof. Let an element $((1-\xi) r t+\xi R, \rho r, \xi a)$ belongs to $\mathbb{M}_{4}$. Then it satisfies the equality given in (4.1). Hence, we have

$$
2 r^{2} \rho^{2}=\frac{\left(\xi^{2}\left[R^{2}+r^{2}-r \sqrt{4 R^{2}+r^{2}}\right]-[\xi(R-r)+r]^{2}\right)^{2}}{\xi^{2}\left[R^{2}+r^{2}-r \sqrt{4 R^{2}+r^{2}}\right]+[\xi(R-r)+r]^{2}} .
$$

Simple but long calculations show that $\rho \in(0,1)$.

\section{PONCELET'S PORISM FOR $n$-GONS} only if

It is proved in [5] that the annulus $C_{r} C_{R}$ have a property of the Poncelet porism for $n$-gons if and where

$$
n B\left(2 \arctan \frac{1}{r} \sqrt{R^{2}-(r-a)^{2}}\right)=B(2 \pi)
$$

$$
B(x)=\int_{0}^{x}\left(1-\left(\frac{r-a \cos s}{R}\right)^{2}\right)^{\frac{-1}{2}} d s .
$$

We define a set $\mathbb{M}_{n}$ as follows: $(R, r, a) \in \mathbb{M}_{n}$ if it satisfies the condition (5.1). For $t=\frac{R}{r}$ we have

$$
n B\left(2 \arctan \sqrt{t^{2}-\left(1-\frac{a}{r}\right)^{2}}\right)=B(2 \pi) .
$$

Differentiating (5.3) with respect to $r$ and using (5.2) we obtain 


$$
g\left(t, n, \frac{a}{r}\right) \frac{\partial}{\partial r}\left(\frac{a}{r}\right)=0,
$$

where $g$ is some function.

\section{FINAL REMARKS AND CONCLUSIONS} tions:

In this section we formulate a few open ques-

1. Taking into account the previous sections we conjecture that $\frac{\partial}{\partial r}\left(\frac{a}{r}\right)=0$.

2. How to extend the group acting on $\mathbb{M}$ in such a way that the left action is transitive?

3. Is it possible to repeat all considerations of the Sections 3 and 4 to all known algebraic Fuss formulas for Poncelet porism, see [10].

4. If the answer to the first question is positive then $a$ is a function of two variables $r, t$ of the form $a=r h(t)$, where $h$ is a monotonic function. Find a differential equation for the function $h$.

The solution of the $4^{\text {th }}$ problem gives Fuss formula of the form $a=r h(t)$, where $t=\frac{R}{r}$.

\section{REFERENCES}

1. Berger M.: Geometry. Springer, New York 1994.

2. Bos H.J.M., Kers C., Oort F., Raven D.W.: Poncelet's Closure Theorem. Expos. Math. 5, 1987, 289-364.

3. Cieślak W.: The Poncelet annuli. Beitr. Algebra Geom., 55, 2014, 301-309.

4. Cieślak W., Martini H., Mozgawa W.: On the rotation index of bar billiards and Poncelet's porism. Bull. Belg. Math. Soc. Simon Stevin 20, 2013, 287-300.

5. Cieślak W., Martini H., Mozgawa W.: Rotation indices related to Poncelet's closure theorem. To appear in Ann. Univ. Mariae Curie-Skłodowska.

6. Jacobi C.G.J.: Über die Anwendung der elliptischen Transcendenten auf ein bekanntes Problem der Elementargeometrie. J. Reine Angew. Math 3, $1823,376-387$.

7. Schoenberg I.J.: On Jacobi-Bertrand's proof of a Theorem of Poncelet. Studies in Pure Mathematics to the Memory of Paul Turan, Basel, Switzerland, Birkhäuser, 1983, 623-627.

8. Tabachnikov S.: Poncelet's Theorem and Dual Billards. Enseign. Math. 39, 1993, 189-194.

9. Tabachnikov S.: Geometry and Billiards. Amer. Math. Soc., Providence, RI, 2005.

10. Weisstein E.W.: Poncelet's Porism. In: Math World - A Wolfram Web Resource. http://mathworld.wolfram.com/PonceletsPorism.html 